of the same species, is not to be entirely overlooked, even though it be highly improbable. The discovery of such appendages on a Trichopterous larva would, indeed, be sufficiently surprising. The gills of Trichopterous larvae are simple or tufted filaments; and the larvae of the Hydroptilidae, so far as known, do not possess even these. Stout processes, articulated at the sides of the abdomen and arranged segmentally are entirely unknown in this order, but occur in certain Coleopterous and Neuropterous larvae. Most like the appendages of our prepupa are the lateral filaments of the larvae of Sialidae, especially, of Sialis: here the several jointed lateral processes are articulated to the sides of the abdomen. In Sisyra and Climacia (family Hemerobiidae of Neuroptera) are similar, jointed filaments directed toward the midventral line beneath the abdomen.

I am inclined to believe that these appendages are inherited from some remote, primitive Neuropterous type. I regard them as belonging in the same category as the large, transitory mandibles of the pupa.

\title{
RECORDS OF THE HABITS OF NEW MEXICAN COLEOP'TERA.
}

BY T. D. A. COCKERELL, EAST LAS VEGAS, N. MEX.

Unless the contrary is specified, the authority for the identification is in every case Mr. H. C. Fall, to whom I am under the greatest obligations. When the collector's name is not given, the material was collected by the present writer. Townsend $=$ C. H. T. Townsend. Wooton $=$ E. O. Wooton.

Cicindela sperata Lec. Rincon, July 5 ; numerous in the bed of the Rio Grande, copulating.

Hippodamia sinuata Muls. Mescalero, on Chrysothamnus graveolens glabratus, Oct. I.

Coccinella oculata Fab. Mescalero, on Chrysothamnus graveolens glabratus, Oct. 2 .
Psyllobora 20-maculata Say. Rio Ruidoso, about $6500 \mathrm{ft}$., on Rhus glabra, July 24. (Townsend).

Chrysobothris carinipennis Lec. Rio Ruidoso, about $6_{500} \mathrm{ft}$., on cut pine branches, Aug. 4. (Townsend).

Chrysobothris debilis Lec. (det. Wickham). In coitu on Prosopis glandulosa, May I3, I892. (Townsend).

Acmeodera sparsa Horn. Organ Mts., back of S. Augustine, on Chrysopsis villosa. Sept. I. (Wooton); Rio Ruidoso, about $6500 \mathrm{ft}$., on flowers of Achillea millefolium, July 30 . (Townsend).

A. disjuncta Fall. La Cueva, Organ Mts. (Townsend). I collected one at 
Juarez, State of Chihuahua, Mexico, Oct. 6, on Helianthus annuus.

A. scalaris Mann. Sand hills near Mesilla Park on flowers of Polypteris hookeriana, Sept. 15 (Townsend).

Lygistopterus rubripennis Lec. Rio Ruidoso, about $6900 \mathrm{ft}$., on flowers of Verbascum thapsus, July 20. (Townsend).

Chauliognathus limbicollis Lec. Above Mescalero, on Spharalcea fendleri, Aug. 2 I. (Wooton).

Macrodactylus uniformis Horn. Rio Ruidoso, about $6500 \mathrm{ft}$. on flowers of Monarda stricta, July 18 . (Townsend); Lone Mtn., July 6, on Concord grape vine.

Rhopalophora laevicollis Lec. Rio Ruidoso, about $6600 \mathrm{ft}$., on flowers of Solidago trinervata, July 20 . (Townsend).

Tragidion fulvipenne Say. Dripping Spring, Organ Mts., several on a Kermesinfested oak branch.

Tylosis maculata Lec. La Cueva, Organ Mts., about $5300 \mathrm{ft}$., on leaves of Sphaeralcea, Aug. 31. (Townsend). This and the next both have the colors of the flowers of the plants on which they occur.

Crossidius pulchellus Lec. Mescalero, Oct. 2, on Chrysothamnus graveolens glabratus; also from Mesilla Park (det. Wickham), on Gutierrezia glomerella, Sept. 23.

Strangalia sexnotata Hald. Rio Ruidoso, about $6600 \mathrm{ft}$., on flowers of Solidago trinervata, July $20 . \quad$ (Townsend). Leptura canadensis cribripennis Lec.
Rio Ruidoso, on flowers of Rhus glabra, July 2 I (Townsend).

Lema concolor Lec. S. Fork Eagle Creek, about $8000 \mathrm{ft}$., on brake fern, Aug. 13. (Townsend).

L. trilineata Oliv. Mesilla Park, on cultivated Datura, Aug. 5.

Coscinoptera axillaris Lec. La Cueva, Organ Mts., on flowers of Fallugia, Sept. 3. (Townsend).

Chlamy's plicata Fabr. (det. Horn). Mesilla Park, on Larrea.

Cryptocephalus spurcus Lec. Mesilla Park, on Pliuhea borealis, May i4. Also occurs on Larrea.

C. quadrimaculatus Say. Rio Ruidoso, about $7000 \mathrm{ft}$, , on brake fern, Aug. 6 . (Townsend).

Calligrapha serpentina Rog. San Augustine Ranch, on Sphaeralcea munroana, Sept. I. (Wooton).

Microrhopala vittata Fab. Rio Ruidoso, about $7000 \mathrm{ft}$., on brake fern, Aug. 6. (Townsend).

Coptocycla clavata Fab. Mesilla, Aug. I8, on Physalis.

C. aurichalcea Fab. Rio Ruidoso, about $6500 \mathrm{ft}$., on Rhus glabra, July 24. ('Townsend).

Bruchus amicus Horn. La Cueva, Organ Mts., about $5300 \mathrm{ft}$., on flowers of Lippia rerightii, Sept. 5. (Townsend).

B. seminulum Horn. Las Vegas, on flowers of Petalostemon oligophyllus, July, $2 \mathrm{I}$.

Epitragus canaliculatus Say. Santa Fé, "eating blackberries," August. (Myrtle Boyle).

Statira pluripunctata, Horn. San 
Augustine Ranch, in flowers of Datura meteloides.

Mordella marginata Mels. Rio Ruidoso, on flowers of Rhus glabra, July i 9, about $6500 \mathrm{ft}$. (Townsend).

Zonitis Alavida Lec. Las Vegas, at flowers of Cleome serrulata, June 29.

Epicauta pennsylvanica DeGeer. Above Mescalero, on Sphaeralcea fendleri, Aug. 2 I. (Wooton)*

Eupompha fissiceps Lec. (det. Schwarz). Plains east of San Andreas Mts., on

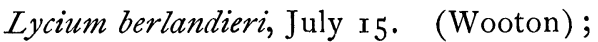
Mesilla Park, July r 7, one on Larrea.

Rhipiphorus cruentus Germ. Fillmore Cañon, Organ Mts., on flowers of Solidago canadensis arizonica, Sept. I, about $5700 \mathrm{ft}$. (Townsend).

Attelabus bipustulatus Fab. Rio Ruidoso, about $6500 \mathrm{ft}$., on Rhus glabra, July 24. (Townsend).

Peritaxia hispida, Horn. La Cueva, Organ Mts., under prostrate sotol, Sept. 4. (Townsend). The sotol is Dasylirion wheeleri.

Pandeletejus cinereus Horn. Las Cruces, on Prosopis glandulosa, May 6, (Townsend).

Apion tenuirostrum Sm. and $A$. varicorne Sm., together on flowers of Petalostemon oligophyllus, Las Vegas, July $2 \mathrm{r}$.

Smicronyx imbricatus Csy. Las Vegas, on flowers of Convolvulus arvensis, June, I 7 .

Otidocephalus vittatus Horn. Embudo,

\footnotetext{
* I may as well record here the occurrence of Epicauta caviceps Horn, and E. wheeleri Ulke, (both det. Wickliam) on Isocoma hartwegi at Tuscon, Arizona, the specimens received from Prof. Toumey.
}

on Chrysothamnus, Sept. 26.

Anthonomus albopilosus Dietz. Embudo, on Croton, Sept. 25.

Desmoglyptus crenatus Lec. Lone Mtn., on Concord grape vine, June 6.

Trichobaris compacta Csy. La Cueva, Organ Mts., on leaves of Datura meteloides, Aug. 3o. (Townsend).

Centrinus acuminatus Csy. Las Cruces, on flowers of Sphaeralcea Sept. 9. (Townsend).

Scyphophorus acupunctatus Gyll. La Cueva, Organ Mts., under prostrate sotol, Sept. 3. (Townsend).

Pityophagus verticalis Horn. Eagle Creek, about $7000 \mathrm{ft}$., Aug. II, in hole in live pine at Gilmore's Ranch. (Townsend.)

Dendroctonus terebrans Oliv. Eagle Creek, about $7000 \mathrm{ft}$, Aug. I I. Townsend has written the following note: "Gilmore's Ranch; boring in live pine tree roots between bark and sapwood and making hole through resin both hard and soft; keeping hole open to outside; resin is running continually. When resin gets hard beetle bores out, leaving pile of chips of resin marking hole. (Alfred Holt)."

\section{Dichopetala BRevicauda - A Cor- RECTION.}

Under this name Scudder described (Can. ent., xxxii, 331-332, I900) a locustid from California and more recently through inadvertence proposed the same name for a species from New Mexico (First list orth. New Mex., p. 

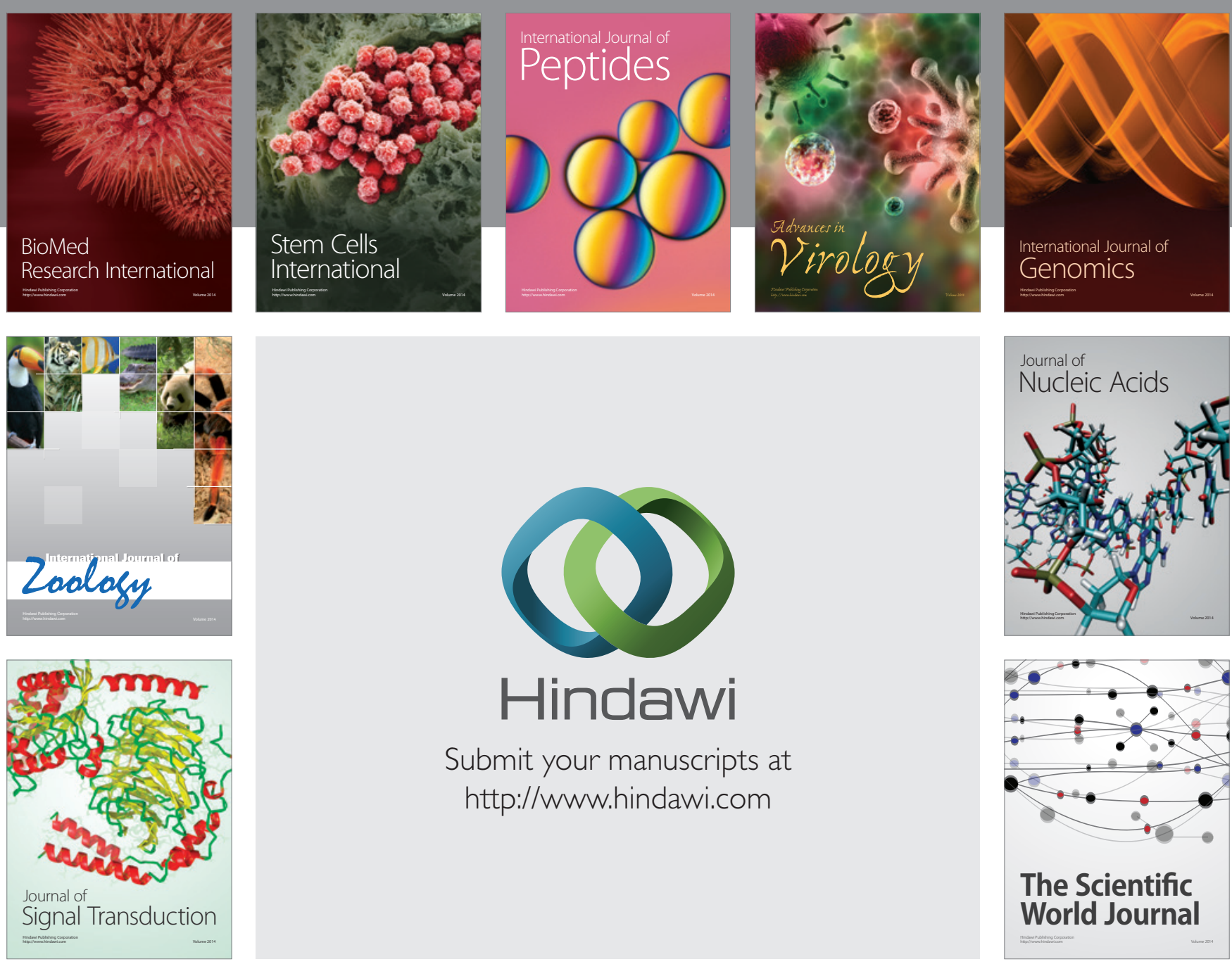

Submit your manuscripts at

http://www.hindawi.com
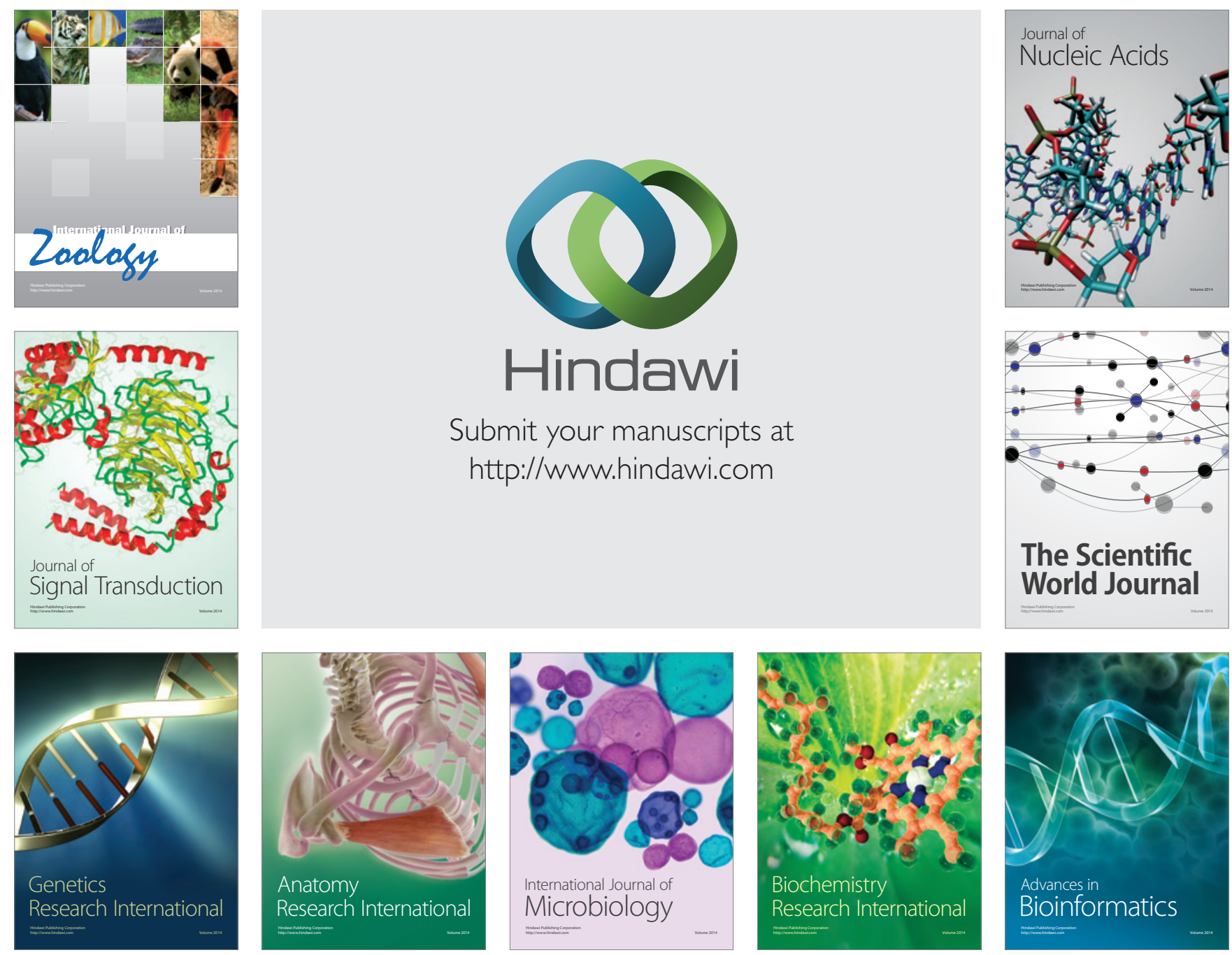

The Scientific World Journal
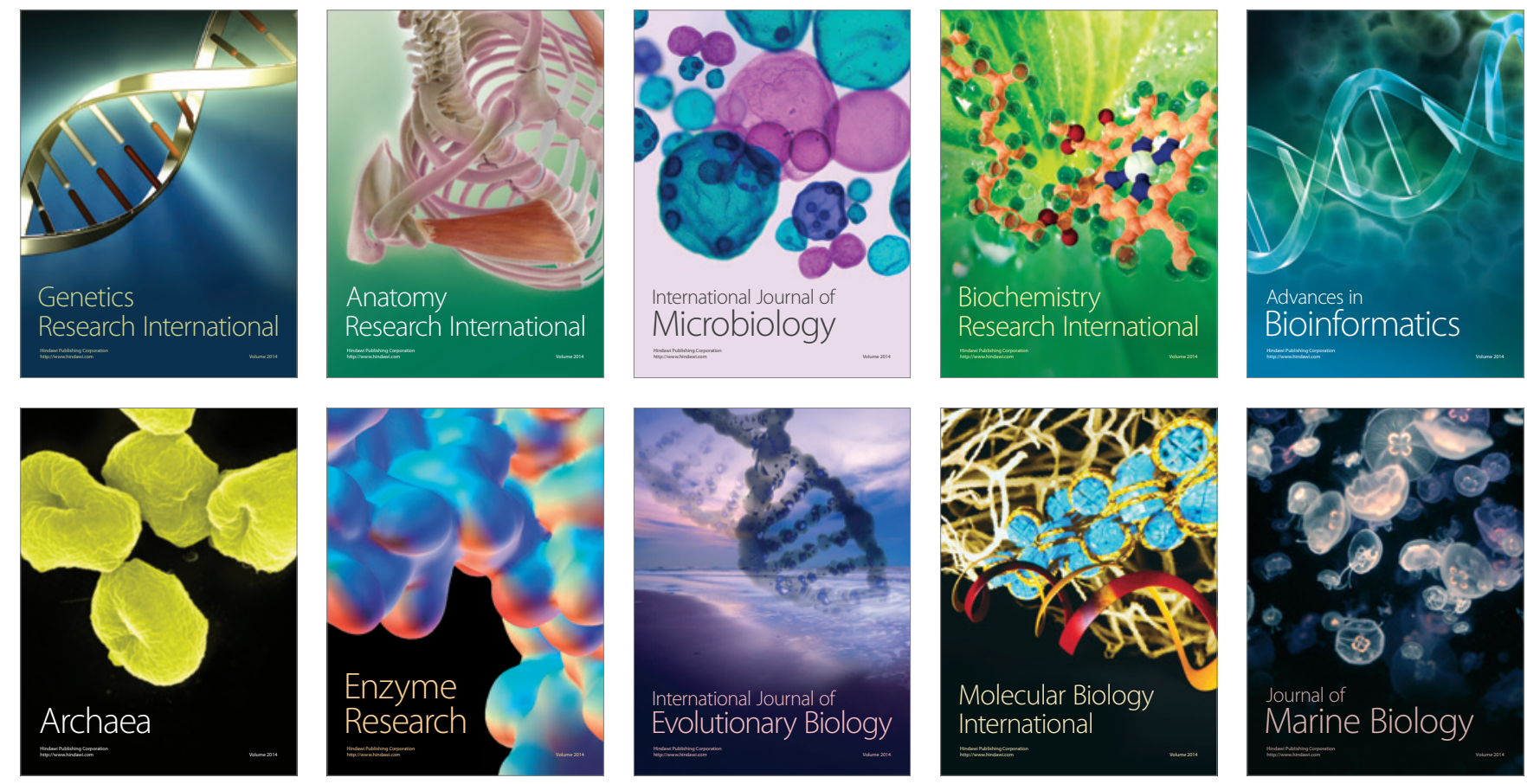